\title{
Population Growth Due to Earlier Childbearing
}

\section{I-Chun Chen}

School of International Studies, Kwansei Gakuin University, Hyogo, Japan

Email: frauleinchen77@gmail.com

How to cite this paper: Chen, I-C. (2020). Population Growth Due to Earlier Childbearing. Modern Economy, 11, 1966-1975. https://doi.org/10.4236/me.2020.1111131

Received: October 5, 2020

Accepted: November 27, 2020

Published: November 30, 2020

Copyright $\odot 2020$ by author(s) and Scientific Research Publishing Inc. This work is licensed under the Creative Commons Attribution International License (CC BY 4.0).

http://creativecommons.org/licenses/by/4.0/

\begin{abstract}
This paper analyzed the effects of earlier childbearing on the population. Earlier childbearing results in the increase in the total fertility rate due to several effects, the tempo effect, baby boom effect, and echo effect. Moreover, the population can increase as many generations live in the same period. In this paper, I proposed to increase the total population by changing the childbearing behavior, which is to bring the age at childbearing forward to younger age. Although the total population is still declining in the long run, the earlier childbearing behavior slows down the speed of declining population.
\end{abstract}

\section{Keywords}

Population, Total Fertility Rate, Age at Childbearing

\section{Introduction}

Over the past decades, the total fertility rate (TFR), has been declining in many countries. Especially, from 1970s, the total fertility rate stood below the replacement rate (2.1) that required for population replacement in most developed countries (see Figure 1). Also, the reduction in population is viewed problematic in some countries. Although a shrinking population is caused by several factors, many discussions indicate that prolonged low total fertility rate mainly attributed to this phenomenon.

A direct cause of the decline in the total fertility rate is the quantum effect, which is a reduction in the number of births a woman has in her lifetime. In addition, if there is an increase in the mean age of childbearing but without affecting the total number of children a woman has over the course of their lives, the number of births in each year will decrease, which is called the tempo effect. However, even there is a tempo effect because of the later childbearing, eventually total population would keep constant if there is no change on the number of children per woman has in her life time (Bongaarts \& Feeney, 1998). 
6

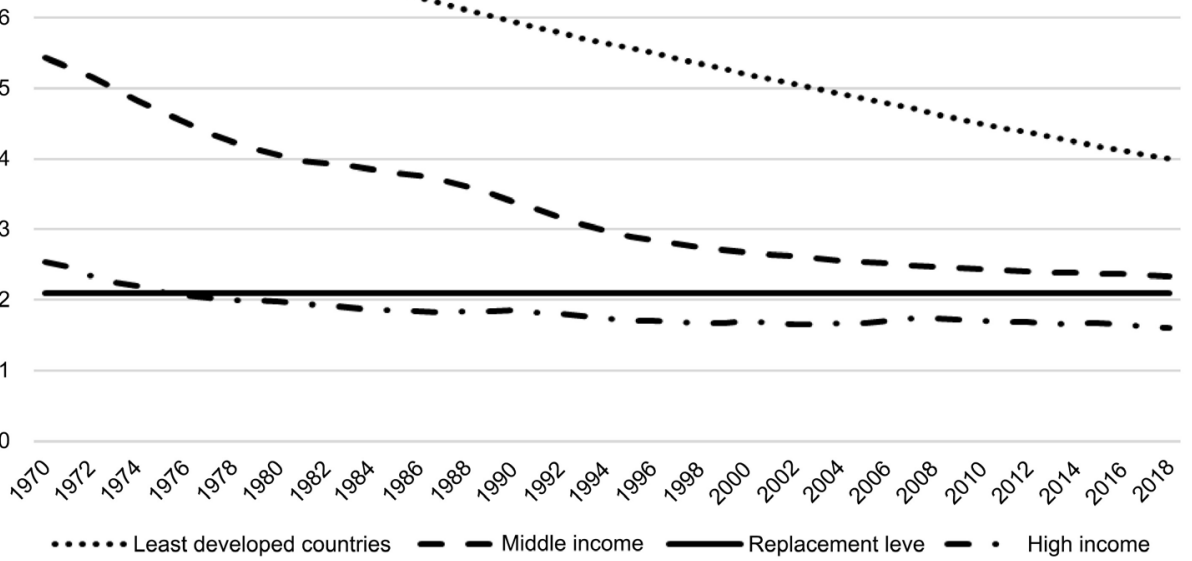

Figure 1. Total fertility rate by countries. Source: World Development Indicators, World Bank.

Women who delayed the timing of childbearing may influence the total fertility by decreasing the number of children they have over their life. And that is known as tempo-quantum interaction effect.

It has been suggested to increase total fertility rate by policy interventions. Some papers have suggested increasing total fertility rate through quantum effect, which is to increase the number of children a woman has. Some papers have focused on the tempo effect. In Europe, there are extensive discussions on the tempo effect playing key role in driving the indicators of fertility and marriage into low level (Bongaarts \& Feeney, 1998; Sobotka, 2004). Yoo \& Sobotka (2018) also stated that the tempo effect has had a strong and persistent negative influence on period TFRs in Korea since the early 1980s. And there were upturns in the period TFR in Europe between 1998 and 2008, which could be largely explained by a decline in the pace of fertility postponement (Bongaarts \& Sobotka, 2012). The policies aiming to end the delayed or even to advance childbearing time would have significant long-term moderating effects on population decline (Lutz et al., 2003; Goldstein et al., 2003). In other words, the changes in childbearing timing can affect the total fertility rate and given quantum effect is fixed. Hence, the changes in the childbearing behavior could affect the level of fertility rate and further the population.

In this paper, I focus on the effects of earlier childbearing on the population. It shows that even if the quantum effect is fixed, the total fertility rate will temporarily increase because of baby boom effects caused by earlier childbearing. Once there is a baby boom, the number of children will increase because of the demographic echo of the baby boomers, which is also called echo effect. Earlier childbearing results in the increase in the total fertility rate due to several effects, the tempo effect, baby boom effect, and echo effect. That will contribute to the population increase. Moreover, the population can increase as many generations live 
in the same period. However, if the total fertility rate is too low, the population still cannot increase. But the pace of declining population can be reduced.

Also, the analytical examination using a simple model and numerical calculations is applied to current demographic situation in Japan. Japan is a country not only experiencing the low TFR but also population declining. Hence, this paper can provide an insight that the earlier childbearing behavior contributes to mitigate the problem of population decline in the aging economy.

This article proceeds as follows. Section 2 presents a simple calculation using an overlapping generation model and it contrasts the number of births and the total population when the childbearing behavior changes. Section 3 provides numerical examples. Sections 4 concludes.

\section{Model}

\subsection{Basic and Earlier Childbearing Scenarios}

Assume that each person lives in the society for $L$ periods and has $f$ children in the $d^{\text {th }}$ period of his/her life. If $0<f \leq 1$, it means there is low fertility in this society. It means there will be shrinking population and aging in this society. People born in the society period $t-d+1$ bear child in the society period $t$. Then their child begins $1^{\text {st }}$ period of the life in the $t^{\text {th }}$ society period. In addition, assuming the number of children each person has is constant in each generation. Hence, the number of births in society period $t, N C_{t}$, is as follows,

$$
N C_{t}=f N C_{t-d+1}=f^{\frac{t}{d-1}} N C_{0}
$$

where $N C_{0}$ is the number of the births in 0 society period.

In the society period $t$, people born from society period $t-L+1$ to $t$ are alive. So, the total population in the $t^{\text {th }}$ society period, $P_{t}$, is as follows,

$$
P_{t}=\sum_{i=t-L+1}^{t} N C_{i}=N C_{0} \sum_{i=t-L+1}^{t} f^{\frac{i}{d-1}}
$$

This is basic scenario.

Next, considering an earlier childbearing scenario, each person gives birth in the $s^{\text {th }}$ period of his/her life ( $d>s$, i.e., $d-s$ periods earlier to bear the child) from the society period $T$. Besides, there are no changes on life expectancy and the quantum of fertility for each generation in this society.

People change their childbearing behavior and advance to give birth at $s^{\text {th }}$ period of their life from society period $T$. Hence, except from people born in $T-S$ $+1^{\text {th }}$ society period, who give birth in the society period $T$ by schedule, people born between society period $t-d+1$ and $t-s$ will also bear the child at the same society period. Therefore, in the society period $T$, there is a baby boom due to the changes in the childbearing behavior. In the society period $T$, the total population increases, that is baby boom effect.

In addition, baby boomers will occur not only in $T^{\text {th }}$ society period but also in the society periods $T+z s$ ( $z$ is integer, and $z \geq 0$ ) because every $s$ periods there will be another baby boom happened. The offspring of baby boomers will also 
have their child and thus cause another baby boom. Hence, the number of births in the periods $T+z s$ is as follows

$$
N C_{T+z s}=f^{z+1} \sum_{i=T-d+1}^{T-s+1} N C_{i}=N C_{0} f^{z+1} \sum_{i=T-d+1}^{T-s+1} f^{\frac{i}{d-1}}
$$

Since the baby booms will only occur every $s$ periods. Hence, the increase in the total population in those periods is due to the echo effect. In periods without the baby boom, the number of births in the society periods $T+z s+u,(z \geq 0,1 \leq u \leq$ $s-1)$ is as follows,

$$
N C_{T+z s+u}=f^{z+1} N C_{T+(z-1) s+u}=f^{z+1} f^{\frac{T \square s+u}{d-1}} N C_{0}
$$

Thus, the number of births rises and falls over the baby boomer generations and the non-baby boomer generations.

Hence, the total population in each period can be expressed as follows,

$$
\begin{gathered}
Q_{y}=\sum_{i=T}^{y} n c_{i}+\sum_{i=T-L+1}^{T-1} c_{i}=\sum_{i=T-v}^{T} n c_{i}+c_{1} \sum_{i=T-L+1}^{T-1} f^{\frac{i}{d-1}} \text { if } y<T+L-1 \\
Q_{y}=\sum_{i=y-L+1}^{y} n c_{i} \text { if } y \geq T+L-1
\end{gathered}
$$

The total population also fluctuates over periods because of the occurrence of baby booms. Note that the number of generations in a society period will increases from $L / d$ to $L / s$ when the childbearing behavior changes from giving birth at $d^{\text {th }}$ period to $s^{\text {th }}$ period of an individual's life. Thus, the number of generations in a society period will increase after $T^{\text {th }}$ society period, and the population will rise due to the baby boom effect and echo effect.

\subsection{A Simple Numerical Example}

First, let $L=3$ and $d=3$, the individual in this society will live for only 3 periods and bear the child in the second period of his/her life. Hence, in the $t^{\text {th }}$ society period, there are people born in the $t^{\text {th }}, t-1^{\text {th }}$ and $t-2^{\text {th }}$ society period alive. From Equation (1), the numbers of births in $t^{\text {th }} t-1^{\text {th }}$ and $t-2^{\text {th }}$ society period are $N C_{b} N C_{t-1}$, and $N C_{t-2}$, respectively. Hence, the number of births in $t^{\text {th }}$ society period is the following,

$$
N C_{t}=f^{\frac{t}{2}} N C_{0}
$$

In addition, the population in the $t^{\text {th }}$ society period consists of 3 generations and is expressed as follows,

$$
P_{t}=N C_{t-2}+N C_{t-1}+N C_{t}=\left(f^{\frac{t}{2}}+f^{\frac{t-1}{2}}+f^{\frac{t-2}{2}}\right) N C_{0}
$$

From $T^{\text {h }}$ society period, assuming there is an earlier childbirth scenario. The timing of childbirth has changed to the second period of life, that is $s=2$. Hence, in society period $T$, those born in the $T-1^{\text {th }}$ and $T-2^{\text {th }}$ society periods will bear the child. Thus, the number of births in $T^{\text {th }}$ society period is the following,

$$
N C_{T}^{c}=f\left(N C_{T-1}+N C_{T-2}\right)=\left(f^{\frac{T}{2}}+f^{\frac{T+1}{2}}\right) N C_{0}
$$


Moreover, from Equation (3a), the number of births in $T+n$ period is as follows.

$$
N C_{T+n}^{c}=f N C_{T+n+1}^{c}=f^{n+1}\left(f^{\frac{T-1}{2}}+f^{\frac{T-2}{2}}\right) N C_{0}
$$

Since $s=2$, there is echo effect in each period after the baby boom occurs. Hence, there is no fluctuation in total population. Thus, the population in the society periods $T^{\text {th }}$ and $T+1^{\text {th }}$ are

$$
P_{T}^{c}=N C_{T}^{c}+N C_{T-1}^{c}+N C_{T-2}^{c}=\left(f^{\frac{T}{2}}+f^{\frac{T+1}{2}}+f^{\frac{T-1}{2}}+f^{\frac{T-2}{2}}\right) N C_{0}
$$

and

$$
P_{T+1}^{c}=N C_{T+1}^{c}+N C_{T}^{c}+N C_{T-1}^{c}=\left(f^{\frac{T-1}{2}}+f^{\frac{T}{2}}+f^{\frac{T+1}{2}}+f^{\frac{T+2}{2}}+f^{\frac{T+3}{2}}\right) N C_{0}
$$

From (4a), the population in the $T+n$ period $(n \geq 2)$ of society is as follows,

$$
P_{T+n}=N C_{T+n}+N C_{T+n-1}+N C_{T+n-2}=\left(f^{n+1}+f^{n}+f^{n-1}\right)\left(f^{\frac{T-1}{2}}+f^{\frac{T-2}{2}}\right) N C_{0}
$$

Next, by comparing the basic and the earlier childbearing scenario, there are four characteristics. First, from Equation (5) and Equation (7), the numbers of births in these two scenarios are compared. In the society period $y=T$, the number of births is greater in the earlier childbearing scenario than in the basic scenario, $N C_{T}^{c}>N C_{T}$. Second, from Equation (6) and Equation (9), the total population in also larger in the earlier childbearing scenario, $P_{T}^{c}>P_{T}$. Therefore, if the childbearing behavior is made at younger age, the number of births will increase and the population will also rise. It should be noted that once the number of births increases and the baby boom occurs, baby boomers in the later society periods also have the same total fertility rate, $f$.

Third, by solving $N C_{T+n^{*}}=N C_{T+n^{*}}^{c}$ from (5) and (8), $n^{*}$ is the value by equaling the number of births in basic and that in earlier childbearing scenario.

$$
n^{*}=\frac{-2 \log \left(f^{\frac{1}{2}}+1\right)}{\log (f)}>0
$$

After advancing the childbearing behavior, alteration of the generation is accelerated by the shorter generation length. Because the fertility rate, $f$, is less than one, the negative intrinsic growth depletes the population growth. Hence, in the long run, the number of births in the earlier childbearing scenario would be less than that in the basic scenario.

Fourth, by solving $P_{T+n^{\prime}}=P_{T+n^{\prime}}^{c}$ from (6) and (11), $n^{\prime}$ is the value that the total population is equal in both basic and younger childbearing scenarios in population in the $t+n^{\prime}$ period of society.

$$
n^{\prime}=\frac{2\left[\log \left(1+f^{-\frac{1}{2}}+f^{-1}\right)-\log \left(1+f^{-1}+f^{-2}\right)-\log \left(f^{\frac{1}{2}}+1\right)\right]}{\log (f)}>0
$$


Note that $n^{\prime}>n^{*}$ is obtained by comparing (12) and (13). Hence, from the above, if the fertility rate is less than one, in the short and medium run, the advancing childbearing behavior would increase the number of births and then rise the total population. However, in the long run, the lower mean generational length would replace one another more quickly and thus decreases the total population. The total population in earlier childbearing scenario will be less than that in the basic scenario.

\section{Numerical Examples}

In this section, I show three numerical simulations to compare the effect of changing childbearing behavior. 1) $N C_{0}=1, L=10, d=6, s=4, T=10$ : Scenario 1 shows the individual lives for 10 periods and bears the children at $6^{\text {th }}$ period of his/her life. And the individual changes the childbearing behavior and has the child at $4^{\text {th }}$ period of his/her life. In addition, with different fertility rate, $f=1.0$, 0.9 , and 0.7 , I will show the differences in total population in the basic case ( $d=$ 6 ) and that in the earlier childbearing scenario $(s=4)$. Figure $2(a)$ shows the transition of the number of births in different fertility rate. It shows the trends of the number of the births are similar in both scenarios. However, the echo effects of changing the childbearing behavior to younger age clearly show in the earlier childbearing scenario. With lower fertility rate, the echo effect is also smaller in the earlier childbearing scenario. Figure 2(b) gives the trends of the total population size in different scenarios and fertility rates. With the fertility rate is 1 , the total population rises with the occurrences of baby booms in the earlier childbearing scenario. With the lower fertility rates, the echo effects become smaller on the total population size in the earlier childbearing scenario. However, comparing to the basic scenario, the total population size is still larger in the younger childbearing scenario.

2) $N C_{0}=1, L=3, d=3, s=2, T=10$ : Figure 3 shows the changes in the population. If $f=1$, the population remains constant in the basic scenario. In the

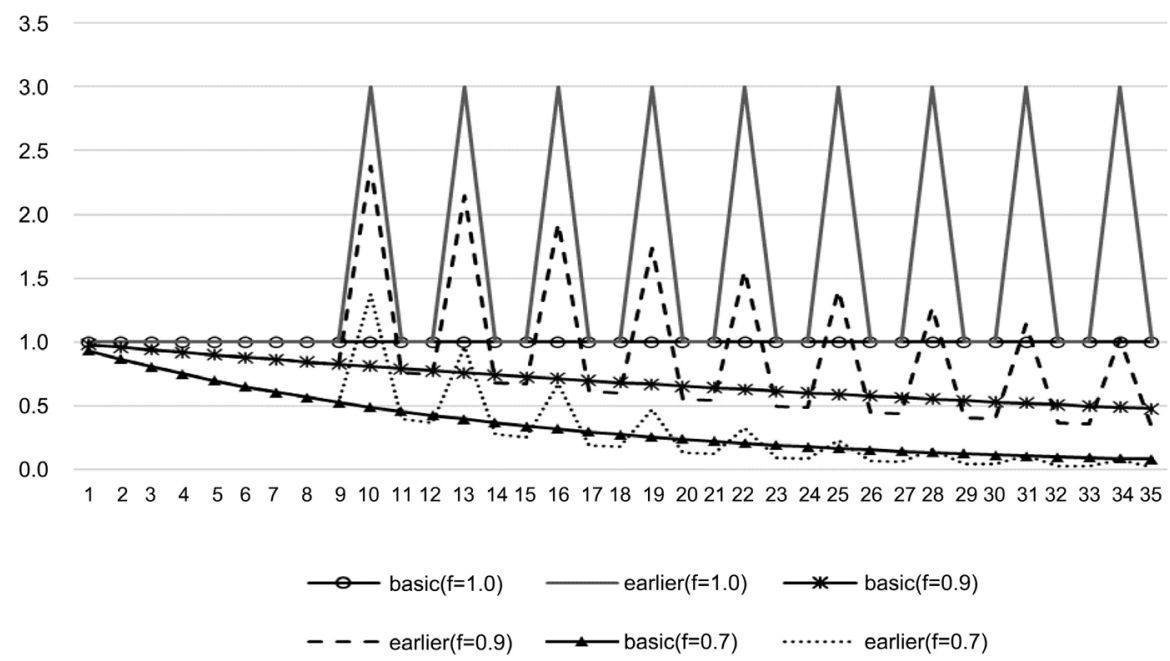

(a) 


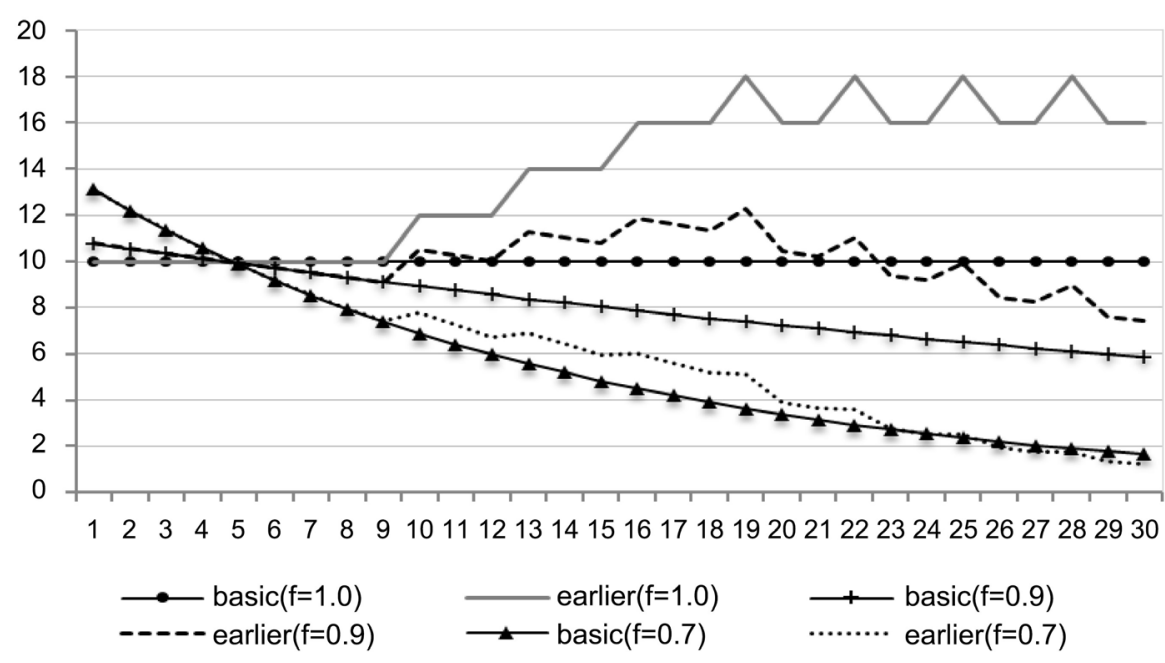

(b)

Figure 2. (a) The number of births under six different scenarios. ( $L=3, d=3, s=2, T=$ 10); (b) Total population under six different scenarios. $(L=10, d=6, s=4, T=10)$

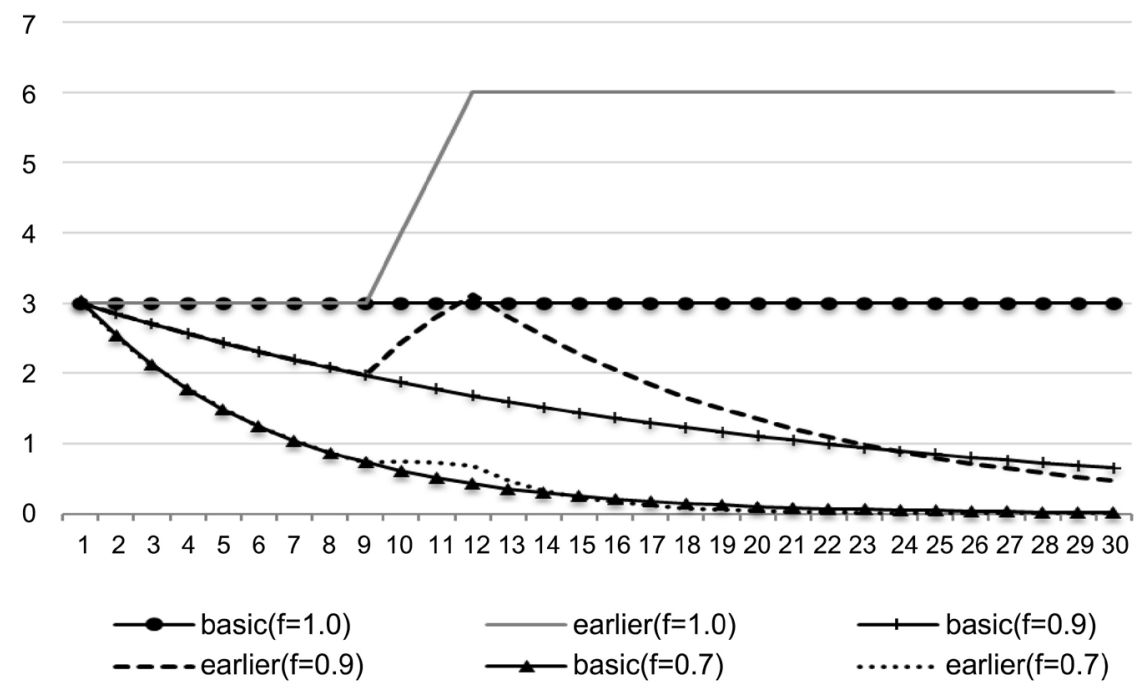

Figure 3. Total population under six different scenarios. $(L=3, d=3, s=2, T=10)$

earlier childbearing scenario, once the age of giving birth becomes younger, the population increases because more generations can survive in the same period. If $f=0.9$, the population in the earlier childbearing scenario temporarily exceeds that in the basic scenario. But over time, the population of baby boomers' descendants continues to decline; eventually it falls below the level in the basic scenario. Hence, from the figures, it shows that the levels of the total population in the earlier childbearing scenario will be lower than those in the basic scenario when $n^{*}=12.7$ and $n^{\prime}=13.7$. If $f=0.7$, the total population in the earlier childbearing scenario also temporarily exceeds that in the basic scenario but at earlier periods, $n^{*}=3.4$ and $n^{\prime}=4.6$, the levels will be lower than those in basic scenario.

3) The calculation refers the Japanese female population as of 2017. The female population is the sum of the number of births in each year up to the aver- 
age age of 87 years old. The age at childbearing is 32-year-old, and the total fertility rate is 0.7 (the half of actual total fertility rate, 1.41). Japan aims to increase the total fertility rate to 1.8 by 2025 . Hence, there will be changes in the number of births from 2025. So, based on the data from Japanese female population in 2017, I will show the changes in the total population under six different scenarios. The six different scenarios are as followings: (i) The basic case: the age at childbearing is 32 ; the total fertility rate is 0.7 . (ii) The age at childbearing is 28 ; the total fertility still keeps at 0.7 . (iii) The age at childbearing is 32 ; the total fertility rate is 0.9 . (iv) The age at childbearing is 28 ; the total fertility rate is 0.9 . (v) The age at childbearing is 32 ; the total fertility rate is 1.0 . (vi) The age at childbearing is 28 ; the total fertility rate is 1.0 .

Figure 4 gives the total population size for these 6 scenarios over a century. The figure clearly shows that if there is no intervention, then the total population will keep declining (case i). However, given the level of total fertility rate, if the age at childbearing was brought forward to 28 -year-old, the size of total population increases first then decreases afterwards (case ii). In other words, the speed of total population decreasing is slower in the earlier childbearing scenario. Moreover, given the levels of the total fertility rate, the positive effects (baby boom effect and echo effects) on the total population in advancing childbearing clearly outweigh that in the basic scenario (case ii, iv, vi). Besides, given the age at childbearing, the higher the total fertility rate, the larger the total population size is.

Figure 5 shows the trends of total population over a shorter period, 15 years. It shows that the total population size at the earlier childbearing scenarios (case ii, iv, and vi) increases at first and then deceases. This clearly confirms the view expressed earlier that the presence of quantum effect makes the total population increases. Hence, the change in the age at childbearing brings about the increase

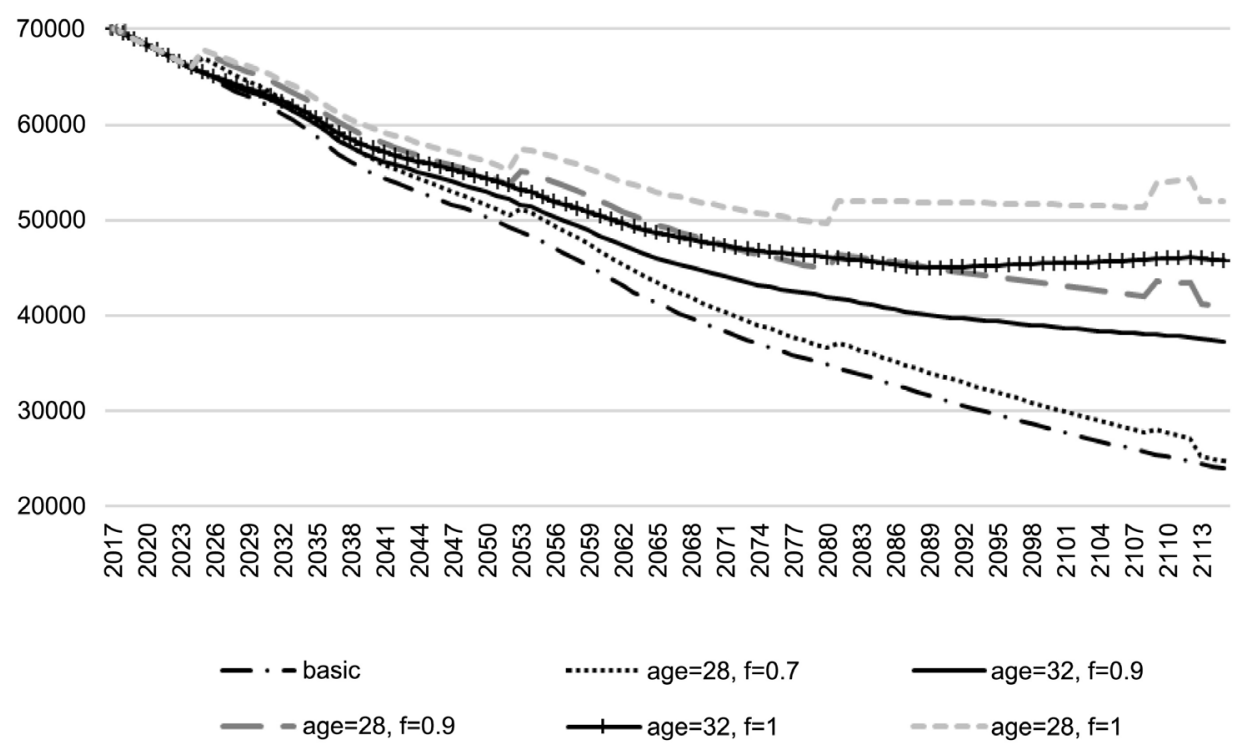

Figure 4. The female population of Japan under six scenarios over one century. 


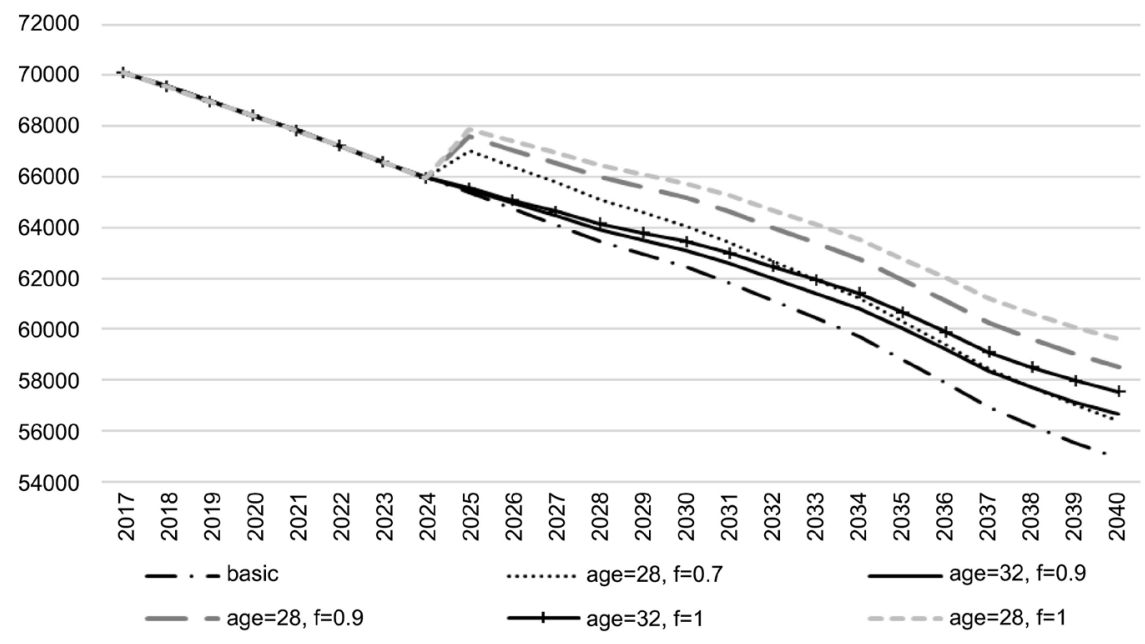

Figure 5. The female population of Japan under six childbearing scenarios over 15 years.

in the total population. But in the long run, the total population size still declines if there is no increase in the total fertility rate.

\section{Discussion and Summary}

The phenomenon of declining fertility in the developed countries has drawn much attention from the policymakers. Many governments in the world have invested heavily in population and family policies to increase the fertility rate but fail to reverse it (Parsons \& Gilmour, 2018; Seo, 2018). Ouedraogo, Tosun, \& Yang (2018) also state there is no significant and robust relationship between change in total fertility rate and pro-fertility ${ }^{1}$ policies using the data from 132 countries. The persistent decrease in fertility leads to aging economy and even population decline in some countries.

In this paper, I propose to increase the total population by changing the childbearing behavior, which is to bring the age at childbearing forward to younger age. Advancing childbearing temporarily could increase the number of births due to tempo, baby boom and echo effects. However, in the long term, the number of births will decrease faster due to the generation length effect. The total population is also affected by those effects. Except from those effects, the effect of overlapping generations also influences the trend of total population. That is, if the age at childbearing is younger, there will be more generations live simultaneously at the same periods. Hence, this results in the increase in the total population.

However, the total population is still declining in the long run. Therefore, to solve the problem, it still needs to increase the total fertility rate itself. Therefore, to encourage giving birth at younger age can be considered as a measure to decrease the pace of declining population. Meanwhile, government could implement the policy to increase the total fertility rate which is the way to solve the ${ }^{1}$ The policies to raise fertility through a variety of government subsidies for childcare and housing, tax incentives, maternal and paternal leave and media campaigns. Ouedraogo, Tosun, \& Yang (2018) refer to it as pro-fertility policy. 
fundamental population problem.

\section{Conflicts of Interest}

The author declares no conflicts of interest regarding the publication of this paper.

\section{References}

Bongaarts, J., \& Feeney, G. (1998). On the Quantum and Tempo of Fertility. Population and Development Review, 24, 271-291. https://doi.org/10.2307/2807974

Bongaarts, J., \& Sobotka, T. (2012). A Demographic Explanation for the Recent Rise in European Fertility. Population and Development Review, 38, 83-120. https://doi.org/10.1111/j.1728-4457.2012.00473.x

Goldstein, J., Lutz, W., \& Scherbov, S. (2003). Long-Term Population Decline in Europe: The Relative Importance of Tempo Effects and Generational Length. Population and Development Review, 29, 699-707. https://doi.org/10.1111/j.1728-4457.2003.00699.x

Lutz, W., O'Neill, B. C., \& Scherbov, S. (2003). Europe's Population at a Turning Point. Science, 299, 1991-1992. https://doi.org/10.1126/science.1080316

Ouedraogo, A., Tosun, M. S., \& Yang, Y. (2018). Fertility and Population Policy. Public Sector Economics, 42, 21-43. https://doi.org/10.3326/pse.42.1.2

Parsons, A. J. Q., \& Gilmour, S. (2018). An Evaluation of Fertility- and Migration-Based Policy Responses to Japan's Ageing Population. PLoS ONE, 13, e0209285. https://doi.org/10.1371/journal.pone.0209285

Seo, S. H. (2018). Low Fertility Trend in the Republic of Korea and the Problems of Its Family and Demographic Policy Implementation. Population and Economics, 3, 29-35. https://doi.org/10.3897/popecon.3.e37938

Sobotka, T. (2004). Is Lowest-Low Fertility in Europe Explained by the Postponement of Childbearing? Population and Development Review, 30, 195-220. https://doi.org/10.1111/j.1728-4457.2004.010 1.x

Yoo, S. H., \& Sobotka, T. (2018). Ultra-Low Fertility in South Korea: The Role of the Tempo Effect. Demographic Research, 38, 549-576.

https://doi.org/10.4054/DemRes.2018.38.22

\section{Appendix}

Table A1. Table of notation.

\begin{tabular}{ll}
\hline Symbol & Meanings \\
\hline$f$ & Number of children each person has \\
$N C_{t}$ & The number of the births in $t^{\text {th }}$ society period \\
$P_{t}$ & The total population in the $t^{\text {th }}$ society period under basic scenario \\
$Q_{y}$ & The total population in the $y^{\text {th }}$ society period under earlier childbearing scenario \\
$L$ & Each individual lives in the society for $L$ periods. \\
$d$ & $\quad$ Each individual has the child at $d$ periods of his/her life under basic scenario \\
$s$ & Each individual has the child at $s$ periods of his/her life under earlier childbearing scenario
\end{tabular}

